\title{
Colmatação e desempenho de sistemas alagados construídos de escoamento horizontal subsuperficial ao longo de oito anos de operação
}

\author{
Clogging and performance of horizontal subsurface \\ flow constructed wetlands over eight years of operation \\ Mateus Pimentel de Matos ${ }^{1 *} \oplus$, Marcos von Sperling ${ }^{2} \oplus$, Antonio Teixeira de Matos $^{2} \oplus$, \\ Daniel Filipe Cristelo Dias ${ }^{2}$, Calebe Rodrigues Soares Santos ${ }^{2}$
}

口

\section{RESUMO}

O presente trabalho objetivou avaliar as condições de funcionamento de dois sistemas alagados construídos de escoamento horizontal subsuperficial (SACs-EHSS), um plantado (SACP) com taboa (Typha latifolia) e outro mantido sem plantas (SACNP), ao longo de oito anos de operação, no tratamento de esgoto sanitário. Mediu-se a extensão do escoamento superficial, como indicativo da colmatação do meio poroso ao longo do tempo, além de diversas variáveis que possibilitem inferências sobre as possíveis perdas de eficiência do sistemana remoção depoluentes dessaáguaresiduária. Asavaliações indicaram que o meio poroso dos dois SACs se encontra colmatado (mais de $80 \%$ do leito apresentando escoamento superficial), estando o SACNP, atualmente, em pior situação, tendo havido prejuízo do seu potencial de remoção de sólidos e nitrogênio em relação ao SACP. Apesar disso, o desempenho em ambos os sistemas permanece satisfatório, não existindo tendência de decréscimo acentuado nas suas capacidades de remoção de poluentes, contrariando a expectativa e corroborando resultados obtidos em trabalhos de monitoramento desses sistemas por longos períodos de tempo. A carga aplicada e o tipo de pré-tratamento a que o esgoto foi submetido parecem ter sido mais decisivos na eficiência dos sistemas em remover nutrientes/poluentes do que o próprio período de operação das unidades, sobretudo no que se refere ao SACP.

Palavras-chave: empolamento; escoamento superficial; macrófitas; remoção de matéria orgânica e nutrientes; tratamento biológico; wetlands construídos.

\begin{abstract}
The objective of this work was to evaluate the conditions of operation of two horizontal subsurface flow constructed wetlands (HSSF-CW), one planted (PCW) with cattail (Typha latifolia), and another, maintained without plants (NPCW), along eight years of operation in sewage treatment. The extent of the overland surface flow was measured as an indicative of the clogging of the pore medium over time, besides several variables that allow to infer possible losses of treatment efficiency in the pollutants removal from wastewater. Evaluations indicated that the porous medium of the two CWs is clogged (more than $80 \%$ of the bed with overland surface flow), and that the NPCW is currently in a worse condition, with a reduction of its solids and nitrogen removal potential in relation to the PCW. Despite this, the performance in both systems remains satisfactory, and there is no tendency of decrease in the capacity to remove pollutants, contrary to expectations, and corroborating the results obtained in works that monitored these systems for long periods of time. The load applied and the type of pretreatment to which the influent was submitted seem to have been more decisive in the systems efficiency in removing nutrients/pollutants than the operating period of the unit, especially for the PCW.
\end{abstract}

Keywords: medium bulking; overland surface flow; macrophytes; organic matter and nutrients removal; biological treatment; constructed wetlands.

\section{INTRODUÇÃO}

Sistemas alagados construídos de escoamento horizontal subsuperficial (SACs-EHSS) são unidades de tratamento de águas residuárias, essencialmente naturais, de grande eficiência na remoção de poluentes. No entanto, à medida que ocorrem os processos físicos (adsorção,

sedimentação e filtração), químicos (precipitação) e biológicos (degradação e absorção) de remoção, há a retenção de sólidos suspensos, formação e crescimento de biofilme, raízes e rizomas, geração de precipitados insolúveis e intemperismo (desgaste) do substrato, os quais concorrem para a obstrução do meio poroso (KADLEC \& WALLACE, 2009).

'Universidade Federal de Lavras - Lavras (MG), Brasil.

¿Universidade Federal Minas Gerais - Belo Horizonte (MG), Brasil.

*Autor correspondente: mateus.matos@deg.ufla.br

Recebido: 13/O2/2017 - Aceito: 11/09/2O17 - Reg. ABES: 175770 
Esse processo contínuo de redução na porosidade, conhecido como colmatação, torna, a princípio, o meio mais restritivo à passagem de particulados, aumentando a eficiência de retenção de sólidos e matéria orgânica particulada nos sistemas. Entretanto, com o avançar do fenômeno, a restrição torna-se um dificultador para a passagem da água residuária, passando o líquido a se movimentar por caminhos menos impedidos e, em estágio mais avançado, a apresentar escoamento superficial, acima do nível do leito. Diante dessas condições, o tempo de detenção hidráulica (TDH) é reduzido, podendo levar ao decréscimo na eficiência dos sistemas (ROUSSEAU; VANROLLEGHEM.; DE PAUW, 2004; ZHANG et al., 2014).

Como a colmatação é um fenômeno que depende das características da água residuária e do substrato (granulometria, uniformidade, formato e tipo de material), das condições de operação (carga aplicada e forma de alimentação), da configuração (direção do escoamento e relação comprimento/largura) do sistema e da presença das espécies vegetais cultivadas, torna-se difícil a predição do tempo necessário para haver colapso de escoamento no sistema. Estudos anteriores apontavam para vida útil entre 50 e 100 anos em SACs-EHSS (CONLEY; DICK; LION, 1991), sendo que a expectativa foi reduzida (WALLACE \& KNIGHT, 2006) conforme novos testes forem conduzidos (NIVALA et al., 2012). Atualmente, há relatos de elevado grau de colmatação depois de dois (PAOLI \& VON SPERLING, 2013) ou quatro anos (CASELLES-OSORIO et al., 2007).

Apesar da expectativa de que haja alterações no funcionamento e eficiência dos SACs, à medida que ocorra a redução na porosidade drenável do leito, não se encontraram, na literatura disponível, muitas pesquisas de monitoramento do desempenho dessas unidades por longos períodos de tempo. Assim, no presente trabalho, objetivou-se monitorar dois sistemas alagados construídos de escoamento horizontal subsuperficial, sendo um plantado com taboa (Typha latifolia) e outro mantido sem plantas, por oito anos, com a finalidade de se observar o efeito da colmatação sobre suas condições operacionais e eficiência na remoção de poluentes.

Torna-se importante ressaltar que, neste estudo, não será abordada uma caracterização mais avançada do processo de colmatação em ambas as unidades, tendo em vista que a alteração na condutividade hidráulica é assunto abordado em Matos et al. (2017) e, na porosidade drenável, em Matos et al. $(2015 ; 2016)$.

\section{MATERIAIS E MÉTODOS}

\section{Área de estudo}

Os testes foram desenvolvidos no Centro de Pesquisa e Treinamento

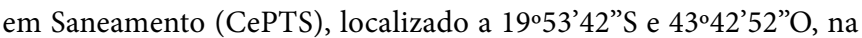
Estação de Tratamento de Esgotos do Arrudas, em Belo Horizonte
(MG). A área tem clima tropical, com temperatura média anual de $22,1^{\circ} \mathrm{C}$ e índice pluviométrico médio de $1.540 \mathrm{~mm}$.ano ${ }^{-1}$.

Os dois SACs-EHSS avaliados estão em operação desde 2007, funcionando em paralelo e recebendo, continuamente, esgoto sanitário submetido a um pré-tratamento, distribuído por meio de uma tubulação perfurada, com uma vazão em torno de $7,5 \mathrm{~m}^{3} \cdot \mathrm{d}^{-1}$ por unidade. Os leitos dos SACs-EHSS foram preenchidos com escória de alto forno, de diâmetro $\mathrm{D}_{10}$ de $19,1 \mathrm{~mm}$, com coeficiente de uniformidade $\left(\mathrm{C}_{\mathrm{U}}\right)$ de 1,2 (DORNELAS; MACHADO; VON SPERLING, 2009). Na Tabela 1, estão apresentados outros aspectos construtivos e operacionais de cada um dos SACs-EHSS. Em um deles (SAC plantado - SACP) foi cultivada a taboa (Typha latifolia), e o outro (SAC não plantado - SACNP) permaneceu sem cultivo.

Como indicativo de colmatação, mediu-se, com o uso de trenas, a extensão do escoamento superficial nos dois SACs.

Dando continuidade às análises realizadas por Dornelas, Machado e Von Sperling (2009), Paoli e von Sperling (2013) e Costa et al. (2015) no mesmo sistema, realizou-se o monitoramento da qualidade do afluente e efluente das unidades. No Quadro 1, estão apresentados os períodos nos quais foram quantificadas essas variáveis, bem como os métodos utilizados para tal, seguindo as recomendações do Standard Methods (APHA; AWWA; WEF; 2012). O período de análises específico deste trabalho, no qual as unidades já apresentavam seu meio poroso intensamente colmatado, ficou compreendido entre outubro de 2013 e julho de 2015, tendo sido feitas, semanalmente, as análises do afluente e efluente dos SACs-EHSS. A não continuidade na análise de algumas variáveis deveu-se ao início tardio ou à interrupção no monitoramento. As medidas de potencial redox em referência ao eletrodo de hidrogênio $\left(E_{\mathrm{H}}\right)$ foram obtidas somando-se $+200 \mathrm{mV}$ aos valores medidos utilizando-se o eletrodo $\mathrm{Ag} / \mathrm{AgCl}$ (potencial redox - POR) (MATOS, 2015).

Como foi objetivo avaliar a capacidade de remoção de poluentes ao longo do tempo, dividiu-se o período de monitoramento dos SACs-EHSS nas suas diferentes configurações de operação (pré-tratamento), inferindo sobre as possíveis alterações na eficiência dentro de cada uma das seguintes três fases:

Tabela 1 - Aspectos construtivos e de projeto de cada sistema alagado construído de escoamento horizontal subsuperficial.

\begin{tabular}{l|c|c}
\hline Variável & Unidade & Valor \\
\hline Altura total (leito) & $\mathrm{m}$ & $\mathrm{0}, 4$ \\
\hline Altura útil (líquido) & $\mathrm{m}$ & $\mathrm{0}, 3$ \\
\hline Comprimento & $\mathrm{m}$ & 25,0 \\
\hline Largura & $\mathrm{m}$ & 3,0 \\
\hline Porosidade do meio filtrante & $\mathrm{m}^{3} \cdot \mathrm{m}^{3}$ & 0,4 \\
\hline Tempo de detenção hidráulica (TDH) teórico & $\mathrm{d}$ & 1,2
\end{tabular}


- Fase 1: SACs recebendo efluente de reator UASB (20 de junho de 2007 a 26 de agosto de 2011);

- Fase 2: SACs recebendo efluente de reator UASB seguido por filtro biológico percolador (FBP) (26 de agosto de 2011 a 01 de novembro de 2013);

- Fase 3: SACs recebendo efluente de reator UASB ( $1^{\circ} \mathrm{de}$ novembro de 2013 a 01 de julho de 2015).

Além do tipo de pré-tratamento, que alterou as cargas aplicadas, o estádio de crescimento das plantas também pode influenciar na eficiência de remoção dos poluentes (KADLEC \& WALLACE, 2009; LEE et al., 2013). A fim de minimizar possíveis interferências dessas etapas do desenvolvimento da taboa e reduzir o efeito de dados discrepantes, dividiram-se as três fases operacionais em subfases (A, B, C, ... I), sendo calculadas as medianas dos dados inseridos em períodos entre cortes. Cada subfase corresponde a um período entre cada corte da parte aérea das plantas. Os cortes foram efetuados em intervalos que variaram de dois a quatro meses.

Por se tratar de dados ambientais, sobretudo de unidades de tratamento de águas residuárias, empregaram-se análises estatísticas não paramétricas, utilizando o software Statistica 8.0 para geração dos testes Kruskal-Wallis, Mann-Whitney e Wilcoxon, respectivamente, para

Quadro 1 - Período e forma de monitoramento de cada variável investigada nos sistemas alagados construídos.

\begin{tabular}{|c|c|c|}
\hline $\begin{array}{l}\text { Período de } \\
\text { monitoramento }\end{array}$ & Variável & Forma de medição \\
\hline 20/09/07 a 10/07/15 & Vazão (Q) & $\begin{array}{l}\text { Medição do volume } \\
\text { coletado por tempo }\end{array}$ \\
\hline 29/03/10 a 10/07/15 & Oxigênio dissolvido (OD) & \multirow{4}{*}{$\begin{array}{c}\text { Sonda } \\
\text { multiparâmetro } \\
\text { HQ4Od }(\mathrm{HACH})\end{array}$} \\
\hline 29/04/11 a 10/07/15 & Potencial redox (POR) & \\
\hline 29/03/10 a 10/07/15 & $\begin{array}{c}\text { Potencial } \\
\text { hidrogeniônico }(\mathrm{pH})\end{array}$ & \\
\hline 14/04/10 a 10/07/15 & $\begin{array}{l}\text { Condutividade } \\
\text { elétrica (CE) }\end{array}$ & \\
\hline 20/09/07 a 10/07/15 & $\begin{array}{l}\text { Sólidos suspensos } \\
\text { totais (SST) }\end{array}$ & \multirow{2}{*}{$\begin{array}{c}\text { Método } \\
\text { gravimétrico }\end{array}$} \\
\hline 20/09/07 a 10/07/15 & $\begin{array}{l}\text { Sólidos suspensos } \\
\text { voláteis (SSV) }\end{array}$ & \\
\hline 20/09/07 a 10/07/15 & $\begin{array}{l}\text { Demanda bioquímica } \\
\text { de oxigênio (DBO) }\end{array}$ & $\begin{array}{l}\text { Oxímetro, } \\
\text { leitura da } \mathrm{DBO}_{5}^{20}\end{array}$ \\
\hline 20/09/07 a 10/07/15 & $\begin{array}{l}\text { Demanda química } \\
\text { de oxigênio (DQO) }\end{array}$ & $\begin{array}{l}\text { Método titulométrico } \\
\text { de refluxo fechado }\end{array}$ \\
\hline 20/09/07 a 10/07/15 & $\begin{array}{l}\text { Nitrogênio total } \\
\text { Kjeldahl (NTK) }\end{array}$ & Método Kjeldahl \\
\hline 20/09/07 a 10/07/15 & Nitratos $\left(\mathrm{NO}_{3}{ }^{-}\right)$ & Cromatografia \\
\hline 20/09/07 a 31/08/11 & Fósforo total (P-total) & $\begin{array}{l}\text { Método molibidílico } \\
\text { e colorimétrico }\end{array}$ \\
\hline 03/01/08 a 10/07/15 & Alcalinidade total (ALT) & Titulométrico \\
\hline
\end{tabular}

comparação de agrupamentos, dados independentes e pareados, sempre com nível de significância de 5\%.

\section{RESULTADOS E DISCUSSÃO}

Aliada à análise de desempenho dos SACs-EHSS, acompanharam-se as alterações visuais na superfície do leito das unidades, monitorando-se a expansão do escoamento superficial da água residuária por meio de medições do seu avanço (Figura 1).

Em 2009, dois anos após o início da operação, Paoli e von Sperling (2013) relataram início de escoamento superficial, tendo maior extensão no SACP. Essa condição manteve-se até ter sido efetuada a troca do reator UASB utilizado no pré-tratamento do esgoto sanitário afluente aos SACs. Essa troca foi necessária devido ao fato de que o reator inicialmente utilizado apresentou problemas de corrosão no seu separador trifásico, o que estava possibilitando a saída de grande quantidade de sólidos desse sistema, proporcionando seu acúmulo no meio poroso dos SACs. Essa colmatação proporcionou condições para que mais de 70\% do SAC apresentasse escoamento superficial do líquido.

A alteração na configuração do sistema, com a substituição do reator UASB e inclusão de um filtro biológico percolador a jusante deste, proporcionou melhorias nas condições de operação dos SACs, passando essas unidades a apresentarem trechos de escoamento superficial visível em torno de apenas $25 \%$ do comprimento dos leitos. Posteriormente, em outra fase operacional do sistema, com a retirada do FBP do processo, houve novo e grande avanço do escoamento superficial, tendo sido alcançado $80 \%$ do comprimento, no SACP, e 90\%, no SACNP. A avaliação feita no dia 31 de março de 2015 foi aquela em que foram observadas as maiores porcentagens de escoamento superficial nos leitos dos SACs, o que provavelmente se deveu à ocorrência de intensa precipitação nesse dia.

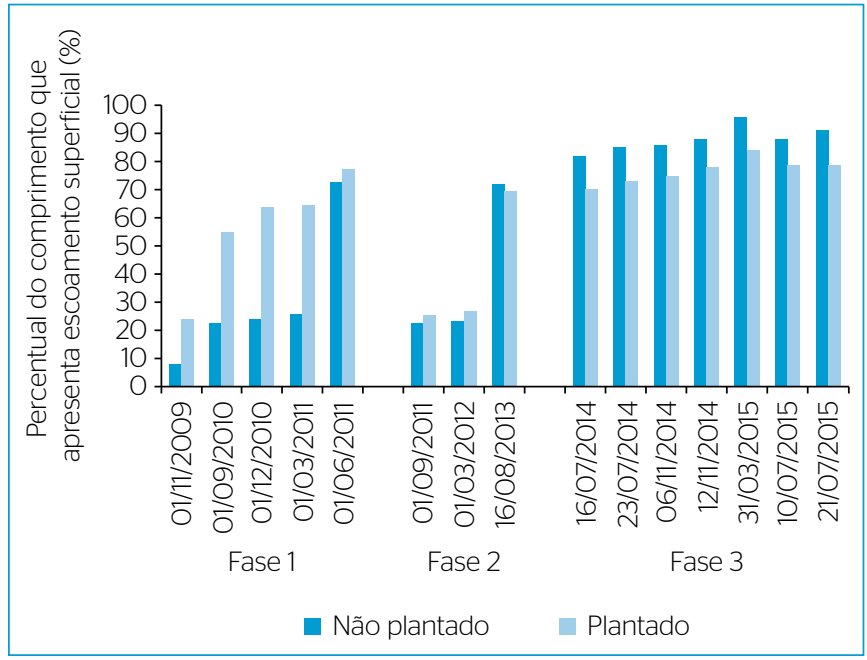

Figura 1 - Monitoramento da extensão do escoamento superficial nos sistemas alagados construídos plantado e não plantado, dado em porcentagem do comprimento das unidades. 
Verificou-se que o SACP se encontrava em um grau de colmatação mais elevado do que o SACNP (PAOLI \& VON SPERLING, 2013), entretanto, com o avançar do tempo, houve atenuação proporcionada pelo desenvolvimento da zona radicular das plantas, como também havia observado Hua et al. (2014) em SACs de escoamento vertical. A explicação pode passar pela ocupação de espaços porosos, sendo o efeito contrabalanceado, posteriormente, pelo mecanismo do empolamento, com expansão do espaço poroso pela penetração e crescimento do sistema radicular (BRIX, 1997; FU et al., 2013; MATOS et al., 2015).

Nas Figuras 2 e 3 estão apresentados os box plots, com os valores médios, mediana, percentis 25 e 75\% e mínimo e máximo de algumas das variáveis medidas na saída dos dois SACs. O teste estatístico Mann Whitney indicou não haver diferença significativa ( $p>5 \%$ ) nos valores medidos no afluente dos SACs, concluindo-se que ambos estavam submetidos às mesmas condições iniciais, podendo se inferir sobre as características do efluente pela comparação entre dados pareados. $\mathrm{Na}$ Tabela 2, estão apresentadas as eficiências de remoção de matéria orgânica, sólidos, nitrogênio e fósforo, com base na carga aplicada.

A alcalinidade da água residuária em tratamento aumentou após o tratamento nos SACs-EHSS, saindo, por exemplo, na primeira fase, de 217 para 255 (SACP) e 249 (SACNP) mg. $L^{-1}$ de $\mathrm{CaCO}_{3}$, na saída das unidades. Possivelmente, a explicação está no contato com o substrato (escória de alto-forno), rico em carbonatos, óxidos e silicatos de Ca e $\mathrm{Mg}$, capazes de neutralizar a acidez e elevar o pH do meio. No entanto, supostamente devido à liberação de ácidos pelas plantas (PAVINATO \&

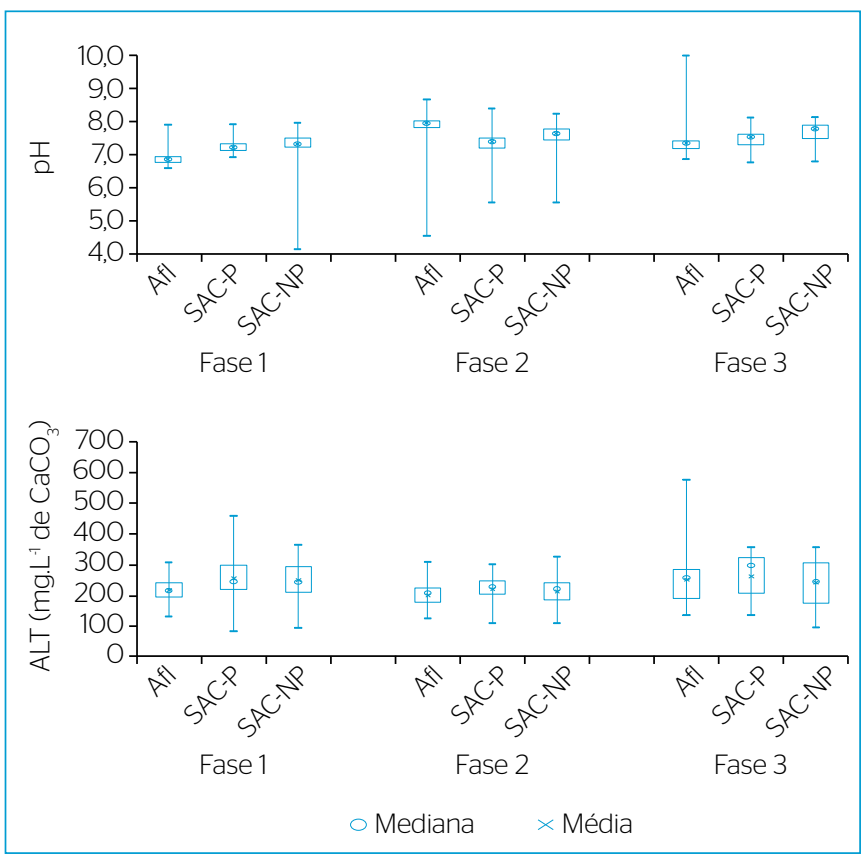

Figura 2 - Box plot dos valores afluente e efluente ( $\mathrm{pH}$ e alcalinidade) dos sistemas alagados construídos, nas três diferentes fases de operação do sistema.
ROSOLEM, 2008) e à absorção de nutrientes das plantas, observou-se menor elevação no $\mathrm{pH}$ do SACP.

A expectativa é de que, após o tratamento, pudesse haver aumento na concentração de oxigênio dissolvido (OD), sobretudo no efluente do SACP, dada à remoção de matéria orgânica e o fornecimento de $\mathrm{O}_{2}$, via aerênquima, pelo sistema radicular (BRIX, 1997). Entretanto, com exceção da Fase 3, em que houve ligeiro aumento, o que ocorreu foi o contrário. Não houve também boa associação entre a concentração de oxigênio e potencial redox $\left(\mathrm{E}_{\mathrm{H}}\right)$. Justificam-se as observações pelo consumo de OD pela microbiota presente em maior número no SACP (GAGNON et al., 2012), onde haveria melhores condições para a conversão da matéria orgânica e também pelo gasto para formação de gel ferro (precipitados) e oxidação do íon amônio. Possivelmente, por essa razão, autores como Matos et al. (2010) também não observaram POR superior em SACP, quando comparado a SACNP, por eles avaliados.

A maior concentração de OD afluente encontrada na Fase 2 se deve à existência de uma fase aeróbia anterior (FBP), proporcionando menor aporte de material orgânico, observado pela demanda bioquímica de oxigênio (DBO), e aeração. Por esse motivo, também se nota maior entrada de nitrato nos dois SACs no período (ver box plots, no item apresentado a seguir).

A condutividade elétrica (CE) aumentou com a passagem do esgoto nos SACs, em razão da mineralização do material orgânico, liberando íons em solução. Pela reconhecida capacidade de extração de nutrientes pelas plantas, esperava-se encontrar menor CE no SACP, o que não foi observado. A explicação para isso está associada às maiores perdas

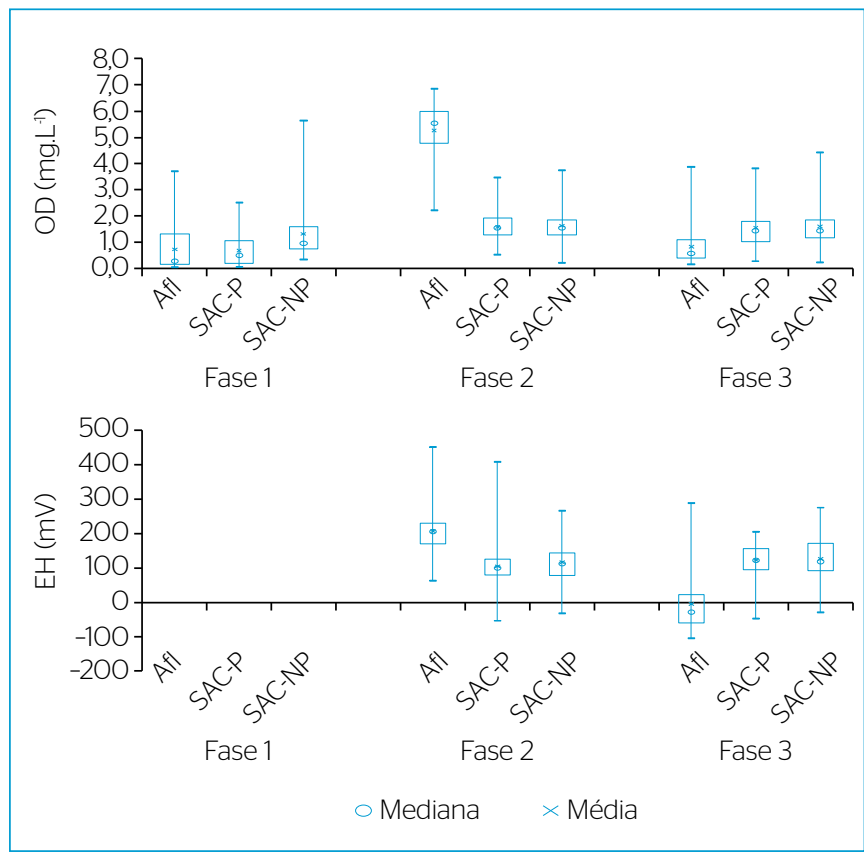

Figura 3 - Box plot dos valores (oxigênio dissolvido e potencial redox $-E_{H}$ ) afluente e efluente dos sistemas alagados construídos, nas três diferentes fases de operação do sistema. 
hídricas ocorrentes nos sistemas plantados que, no caso, se devem à contribuição relativa à transpiração da taboa, o que proporciona maior concentração de sais na água residuária em tratamento nos SACs-EHSS.

Para possibilitar a avaliação do desempenho das unidades, construiu-se a Tabela 2, na qual estão apresentadas as eficiências de remoção calculadas com base nas cargas afluente e efluente, ao invés de simplesmente baseado nas concentrações, o que elimina a interferência dos efeitos de aumento na concentração no efluente, decorrentes das perdas hídricas proporcionadas pela evapotranspiração nesses sistemas. Os dados apresentados são relativos aos valores da mediana da eficiência, já que são esses os que são comparados nos testes estatísticos não paramétricos.

As remoções de sólidos totais foram maiores nas duas primeiras fases no SACNP, da mesma forma que o observado por Tanner, Sukias e Upsdell (1998), o que os autores justificaram ser decorrente da ressuspensão dos sólidos pelas raízes das plantas. Ainda assim, a capacidade de remoção de sólidos suspensos voláteis (SSV) e DBO pelo SACP não foi estatisticamente inferior, indicando que possa haver uma compensação pela maior diversidade da comunidade microbiana hábil para degradação do material orgânico. No balanço, parece haver outra variável, que seriam os sólidos produzidos pelas espécies vegetais (detritos vegetais), o que poderia justificar a equivalência do SACP e SACNP, em relação à remoção de DBO e DQO, apesar do potencial das espécies vegetais em criar um ambiente mais propício para tal (FU et al., 2013). Lee et al. (2004), no entanto, mostram-se mais reticentes quanto ao papel da planta na remoção de sólidos suspensos, afirmando que os processos de retenção nos SACs são meramente físicos. Por outro lado, sabe-se que a malha de raízes pode também contribuir para o crivo do material afluente e para a formação do biofilme (ZHAO; ZHU; TONG, 2009).
Para muitos autores, como Caselles-Osorio et al. (2007), a carga orgânica superficial aplicada é o fator mais importante para a colmatação do meio poroso de SACs, devendo-se observar os valores para não incorrer em risco de aceleração da sua obstrução. A USEPA (2000), por exemplo, sugere uma faixa de 6,0 a 13,3 g.m $\mathrm{m}^{-2} \cdot \mathrm{d}^{-1} \mathrm{de}$ DBO, para ter efetivo tratamento de esgotos domésticos. Em países de clima quente, no tratamento de outras águas residuárias, como as agroindustriais, utilizam-se valores recomendados menos conservadores, de até 50 g.m ${ }^{-2} \cdot \mathrm{d}^{-1}$ de DBO (MATOS \& MATOS, 2017). Garfí et al. (2012) não verificaram escoamento superficial após quatro anos de operação de SACs-EHSS, tendo carga aplicada de $6,0 \mathrm{~g} \cdot \mathrm{m}^{-2} \cdot \mathrm{d}^{-1}$. No entanto, dado o grande número de variáveis envolvidas, a fixação de valores específicos é complexa. Apesar disso, é interessante ter um referencial para inferir sobre a condição de operação do leito. Tendo como base a maior carga aplicada no experimento, que foi de $6,6 \mathrm{~g} \cdot \mathrm{m}^{-2} \cdot \mathrm{d}^{-1} \mathrm{de}$ DBO (mediana) na primeira fase, verifica-se que esta está dentro da faixa para se ter um efetivo tratamento, estando, entretanto, acima do utilizado por Garfí et al. (2012). Esse pode ter sido o motivo para Paoli e von Sperling (2013) terem observado escoamento superficial após apenas dois anos de operação, nesse mesmo sistema.

Outra possibilidade é a observação da carga aplicada na seção transversal (largura $\times$ altura líquida), que é um referencial mais crítico para SACs-EHSS. Kadlec e Wallace (2009) e Nivala et al. (2012) não recomendam a utilização de taxas de aplicação orgânica superiores a 250 g.m $\mathrm{m}^{-2} \cdot \mathrm{d}^{-1}$ de DBO. Considerando a seção transversal de 3,0 m de largura por $0,3 \mathrm{~m}$ de altura do líquido, haveria suplantação em todas as fases, tendo 552 , 259 e 386 g.m . $^{-2} \cdot \mathrm{d}^{-1}$ (medianas), respectivamente nas Fases 1, 2 e 3.

Por algumas vezes, o SACP proporcionou maior remoção de N e P. Porém, como a capacidade da planta de absorver nutrientes é limitada e

Tabela 2 - Mediana das cargas aplicadas e das eficiências de remoção (\%), calculada com base nas cargas afluentes e efluentes, nos dois sistemas alagados construídos de escoamento horizontal subsuperficial, com indicação das diferenças significativas.

\begin{tabular}{|c|c|c|c|c|c|c|c|c|c|}
\hline \multirow{3}{*}{ Variável } & \multicolumn{3}{|c|}{ Fase 1} & \multicolumn{3}{|c|}{ Fase 2} & \multicolumn{3}{|c|}{ Fase 3} \\
\hline & \multirow{2}{*}{$C_{\text {aff. }}\left(g \cdot m^{-2} \cdot d^{-1}\right)$} & \multicolumn{2}{|c|}{ Efic. SAC(\%) } & \multirow{2}{*}{$C_{\text {aft. }}\left(g \cdot m^{-2} \cdot d^{-1}\right)$} & \multicolumn{2}{|c|}{ Efic. SAC(\%) } & \multirow{2}{*}{$C_{\text {aff. }}\left(g \cdot m^{-2} \cdot d^{-1}\right)$} & \multicolumn{2}{|c|}{ Efic. SAC (\%) } \\
\hline & & $\mathrm{P}$ & $\mathrm{NP}$ & & $\mathrm{P}$ & $\mathrm{NP}$ & & $P$ & NP \\
\hline SST & $8,0 a$ & 83 & $88^{*}$ & $5,7 a$ & 82 & $88^{*}$ & $5,6 a$ & 82 & 80 \\
\hline SSV & $2,8 b$ & 91 & 92 & $3,2 b$ & 84 & 88 & 4,8a & 85 & 81 \\
\hline DBO & $6,6 a$ & 77 & 76 & $3,1 \mathrm{~b}$ & 75 & 71 & 4,6a & 80 & 75 \\
\hline DQO & $16,6 a$ & $79^{*}$ & 75 & $10,4 b$ & 76 & 74 & $24,2 a$ & 78 & 66 \\
\hline NTK & $3,5 a$ & 24 & 24 & $3,0 b$ & 36 & 35 & $2,7 \mathrm{~b}$ & $48^{*}$ & 30 \\
\hline $\mathrm{N}-\mathrm{NO}_{3}$ & 0,003ab & -6 & -32 & $0,9 a$ & $98^{*}$ & 97 & $0,2 b$ & -12 & 7 \\
\hline P-Total & 0,3a & $52^{*}$ & 37 & $0,5 b$ & 83 & 85 & - & - & - \\
\hline
\end{tabular}

$\mathrm{C}_{\text {an: }}$ Carga afluente às unidades (g.m².d-1); Efic. SAC (\%): eficiência das unidades plantada (SACP) e não plantada (SACNP); P; plantada; NP: não plantada; SST: sólidos suspensos totais; SSV: sólidos suspensos voláteis; DBO: demanda bioquímica de oxigênio; DQO: demanda química de oxigênio; NTK: nitrogênio total Kjeldahl; *diferença significativa pelo teste de Wilcoxon (significância de 5\%), comparando as eficiências do SACP e SACNP; P-total não foi determinado na Fase 3. NO ${ }_{3}$ tem apenas dois dados de eficiência de carga, por isso não foi realizada a comparação estatística; cargas aplicadas com a mesma letra não diferiram significativamente, entre as diferentes fases, pelo teste de Kruskal-Wallis. 
representa um pequeno percentual da rota de remoção de $\mathrm{N}$ e $\mathrm{P}$, respectivamente 7 e 6\%, segundo Costa et al. (2015), o aumento significativo da carga aplicada de $\mathrm{P}$ da Fase $1\left(0,3 \mathrm{~g} \cdot \mathrm{m}^{-2} \cdot \mathrm{d}^{-1}\right)$ para Fase $2\left(0,5 \mathrm{~g} \cdot \mathrm{m}^{-2} \cdot \mathrm{d}^{-1}\right)$ implicou em diferença não significativa entre o SACP e o SACNP. Por outro lado, a eficiência na remoção global de $\mathrm{P}$ aumentou, o que pode ser atribuído à maior carga aplicada, ao possível desgaste do substrato, com liberação de oxi-hidróxidos de $\mathrm{Fe}$ e $\mathrm{Al}$ para interação com o P presente na água residuária e à maior disponibilidade de pares iônicos para precipitação como sais.

Apesar de não ter sido estatisticamente inferior às obtidas nas demais fases, a menor carga aplicada na Fase 3 também pode ser considerada fator que justificaria as maiores remoções de nitrogênio total Kjeldahl (NTK) no SACP. Como observado na Figura 3, as condições no meio não foram muito favoráveis à nitrificação, a não ser próximo à saída da unidade. Assim, nas Fases 1 e 3, o que pode estar ocorrendo (eficiência negativa) é que não houve tempo hábil para absorção e remoção de $\mathrm{NO}_{3}$ por outros mecanismos (desnitrificação e adsorção), enquanto na Fase 2, boa parte desse se formou no FBP.

Diante dessas observações, reforça-se a importância do pré-tratamento e da carga aplicada, tendo em vista que a capacidade de remoção de nutrientes pelas plantas é baixa. Controle adequado nas condições de operação, ou seja, aporte de matéria orgânica e sólidos em faixas adequadas ao sistema propicia aumento nas eficiências de remoção de poluentes (MATOS et al., 2010) e redução no risco de sua colmatação precoce.

Para inferir sobre o possível efeito da colmatação na eficiência dos sistemas, construíram-se os gráficos box plots, apresentados nas Figuras 4, 5 e 6, nos quais estão apresentadas as três fases subdivididas nas subfases (período entre cortes da parte aérea). Essas subfases foram comparadas pelo teste de Kruskal-Wallis, em nível de 5\% de significância, comparando-se a carga aplicada (letras maiúsculas) e a eficiência de remoção (letras minúsculas). Observa-se que houve alguns poucos registros desses eventos, ocorrendo principalmente na primeira fase, quando os reatores ainda se estabilizavam. Dessa forma, isso indica que, em geral, caso haja verificação de remoções estatisticamente superiores ou inferiores no intervalo, essas poderiam ser atribuídas ao tempo de operação dos sistemas e não às taxas de carregamento.

A análise geral dos box plots apresentados não indica tendência visível de que a eficiência irá diminuir continuamente com o tempo de operação dos SACs-EHSS. Na série da porcentagem de remoção de DBO, apesar da oscilação nos dados, observa-se um princípio de decaimento na capacidade depuradora do sistema, ainda que não significativa, pelo teste Kruskal-Wallis, o que só pode ser confirmado caso haja continuidade no monitoramento desses sistemas.

$\mathrm{Na}$ teoria, a rota de transformações do nitrogênio tende a ser mais influenciada pela colmatação, já que os microrganismos heterotróficos, associados à conversão da matéria carbonácea, têm maior velocidade de reprodução e adaptação às condições do meio, consumindo parte importante do oxigênio dissolvido na água residuária (UDEIGWE \& WANG, 2010). Assim, com o aumento na obstrução dos poros, menores seriam as taxas de trocas gasosas, havendo menor remoção de NTK e menor produção de nitrato (ZHU et al., 2014). No entanto, tal como já discutido, a configuração do pré-tratamento mostrou-se fator de fundamental importância na especiação química de N e sua remoção da água residuária. Além disso, apesar de muitas vezes não se observar diferença significativa entre as cargas aplicadas, as oscilações nas eficiências de remoção parecem estar mais ligadas às variações no aporte de poluentes do que propriamente ao tempo de operação (e grau de colmatação) do sistema.

Com base em todas essas informações, verifica-se que não há comprovação de perda de eficiência nos SACs ao longo dos oito anos de sua operação. Existem alguns indicativos de possível início de subfases em que há decréscimo na eficiência de remoção de $\mathrm{DBO}$, com tendência de queda nas últimas fases do monitoramento, sem, no entanto, proporcionar diferença significativa entre as eficiências obtidas nas subfases (Figura 5). Maior tempo de monitoramento das unidades poderá, no entanto, confirmar possíveis tendências e, conforme já discutido, a eficiência do sistema na remoção de poluentes parece estar mais relacionada à carga aplicada e ao tipo de pré-tratamento do que, propriamente, à colmatação do meio poroso.

Na literatura, há relatos de alguns trabalhos nos quais foi possível observar diminuição na capacidade depuradora dos SACs, como por exemplo, no conduzido por Tanner, Sukias e Upsdell (1998), que relataram redução na remoção de sólidos com o avanço da colmatação. Ranieri e Young (2012) verificaram redução na adsorção de metais pesados, tendo a remoção de níquel diminuído de 83 para $31 \%$ e a de cromo de 79 para 65\%. Porém, apesar de muitos outros autores indicarem a possibilidade de ser a colmatação a responsável pelo colapso do sistema, não há um estudo com longa base de dados que possibilite essa conclusão. As inferências feitas a respeito disso estão baseadas na lógica de que há redução na eficiência em decorrência da diminuição no TDH, o que nem sempre se comprova. No trabalho de Vera et al. (2011), observa-se oscilação comum nas concentrações efluentes de DBO, cumprindo, em quase todos os períodos, a legislação europeia no que se refere ao lançamento de efluentes em corpos hídricos receptores, nos 8 anos de monitoramento de 11 estações de tratamento de águas residuárias, algumas delas sem pós-tratamento. Vymazal (2009) reuniu informações de um período ainda maior, 15 anos de operação de unidades na Polônia, não encontrando, também, evidências de perda de eficiência na remoção de poluentes em SACs-EHSS. Essas evidências podem indicar que a reinfiltração da água residuária em escoamento superficial numa seção não obstruída (WALLACE \& KNIGHT, 2006) e mesmo o funcionamento tal como um SAC de escoamento superficial, possibilitam a manutenção de boas eficiências no sistema. Naz et al. (2009) realizaram um comparativo entre unidades de escoamento subsuperficial horizontal e 
superficial e demonstraram que, apesar da primeira configuração apresentar melhores eficiências, em alguns aspectos, o segundo também proporciona alta capacidade na remoção de poluentes. O acúmulo de material orgânico adsorvente, complexante e quelante (LUEDERITZ et al., 2001) e o desgaste contínuo do meio suporte, proporcionando a disponibilização de sítios ativos de retenção, também são fatores plausíveis de atenuação na perda de eficiência dos sistemas.

Ao final dos oito anos de operação, o SACNP apresenta-se, aparentemente, mais colmatado e, assim, a expectativa seria a de proporcionar menor capacidade na remoção de poluentes do que o SACP. Entretanto, avaliando-se os dados apresentados na Tabela 2, especificamente os da terceira fase, período em que é notada a maior extensão de escoamento superficial visível na unidade sem plantas, não se observa diferença significativa entre os tratamentos, com exceção da variável NTK. A causa pode estar no efeito da colmatação sobre a taboa, que não suportou bem a condição crítica, do trecho próximo à entrada da unidade. Nas zonas mais a jusante, houve maior número de indivíduos vivos, porém, ainda assim, o replantio foi necessário.

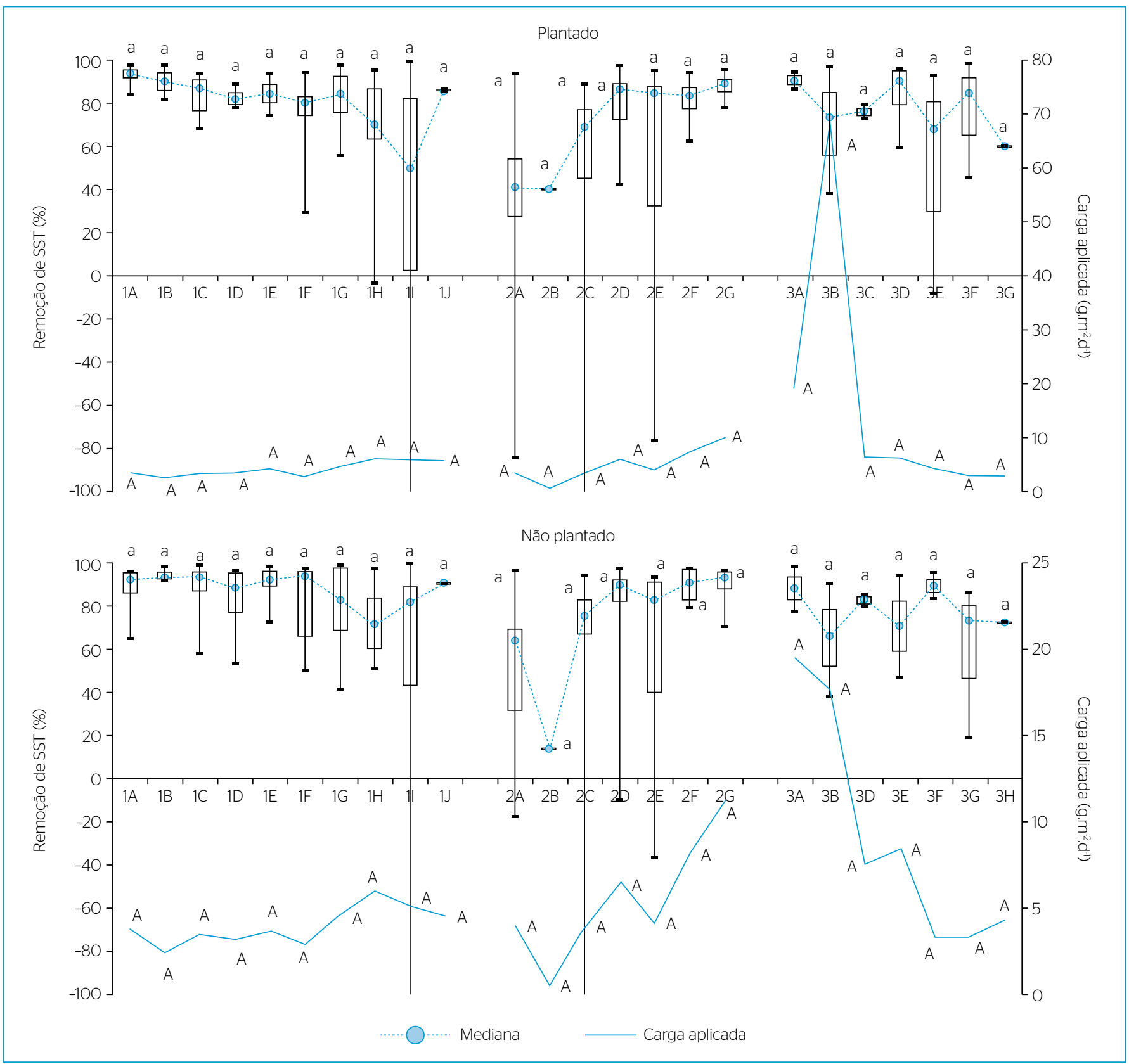

Figura 4 - Box plot das eficiências de remoção de sólidos suspensos totais nos sistemas alagados construídos plantado e não plantado, durante as subfases (períodos entre cortes das plantas) das três fases. Medianas seguidas pela mesma letra não diferem significativamente (minúsculas para mediana e maiúsculas para cargas aplicadas), pelo teste de Kruskal-Wallis, em nível de $5 \%$ de significância. 
Por outro lado, se, nas Fases 1 e 2, a remoção de sólidos suspensos totais (SST) era significativamente maior no SACNP, passou a não haver mais diferença entre os tratamentos na Fase 3, o que pode ser um primeiro indicativo da influência da colmatação do meio poroso nos SACs. De forma análoga, no princípio, as remoções/conversões de nitrogênio na sua forma amoniacal não eram diferentes estatisticamente, no entanto, já na última etapa analisada (Fase 3), observa-se ser a unidade plantada mais efetiva nesse quesito, de acordo com comparação efetuada pelo teste de Wilcoxon.

\section{CONCLUSÕES}

Não foram observados indícios de perda de eficiência na remoção de poluentes com a colmatação, mesmo estando os dois sistemas alagados construídos (SACs) em estágio avançado de obstrução dos poros nos oito anos de monitoramento. Ressalta-se, assim, a robustez das unidades que mantêm as boas eficiências, mesmo em condições críticas de funcionamento. Essas evidências contrariam a expectativa de prejuízo no desempenho das unidades em função da colmatação, corroborando os resultados obtidos em trabalhos nos quais houve o monitoramento

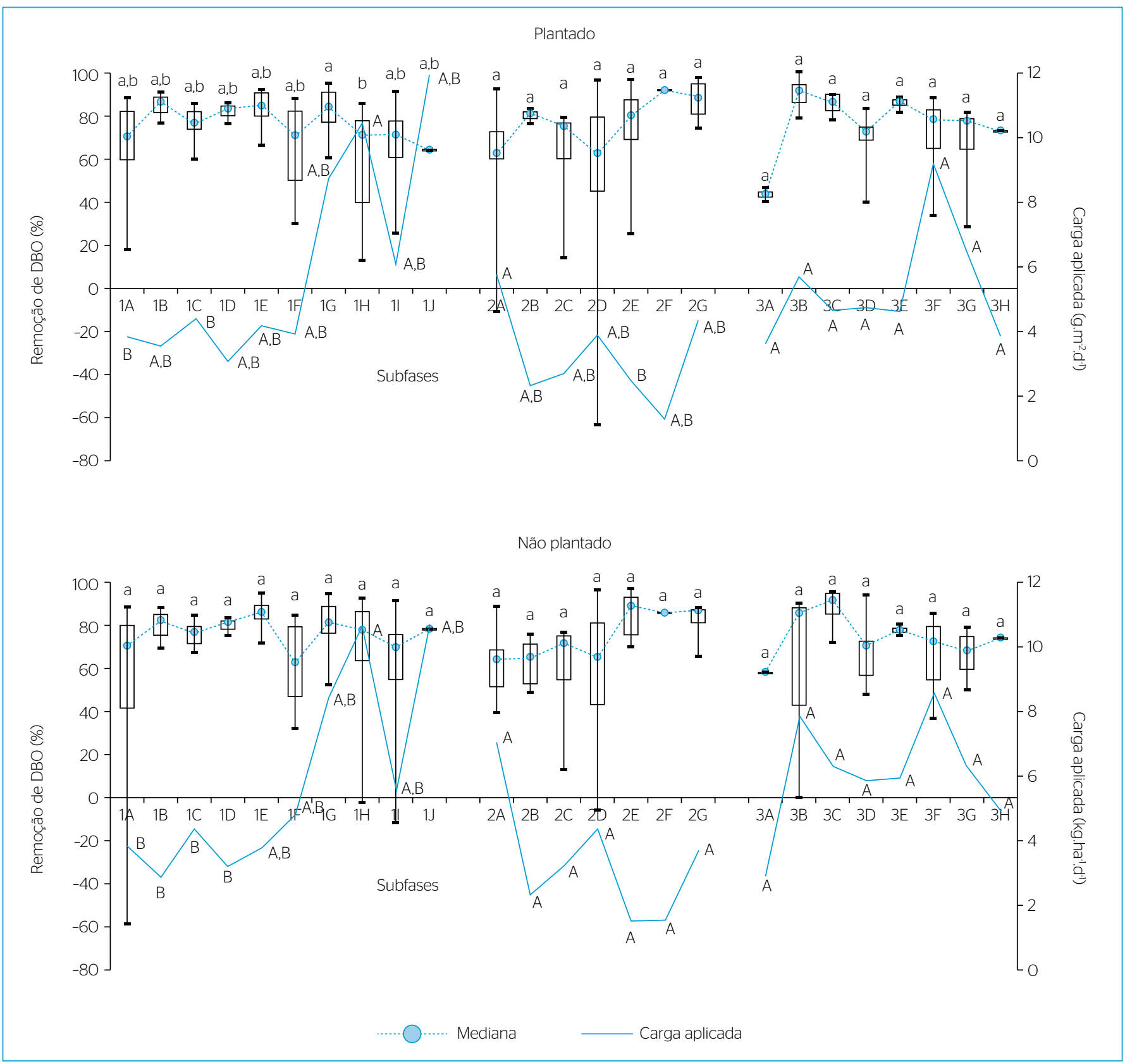

Figura 5 - Box plot das eficiências de remoção de demanda bioquímica de oxigênio nos sistemas alagados construídos plantado e não plantado, durante as subfases (períodos entre cortes das plantas) das três fases. Medianas seguidas pela mesma letra não diferem significativamente (minúsculas para mediana e maiúsculas para cargas aplicadas), pelo teste de Kruskal-Wallis, em nível de $5 \%$ de significância. 


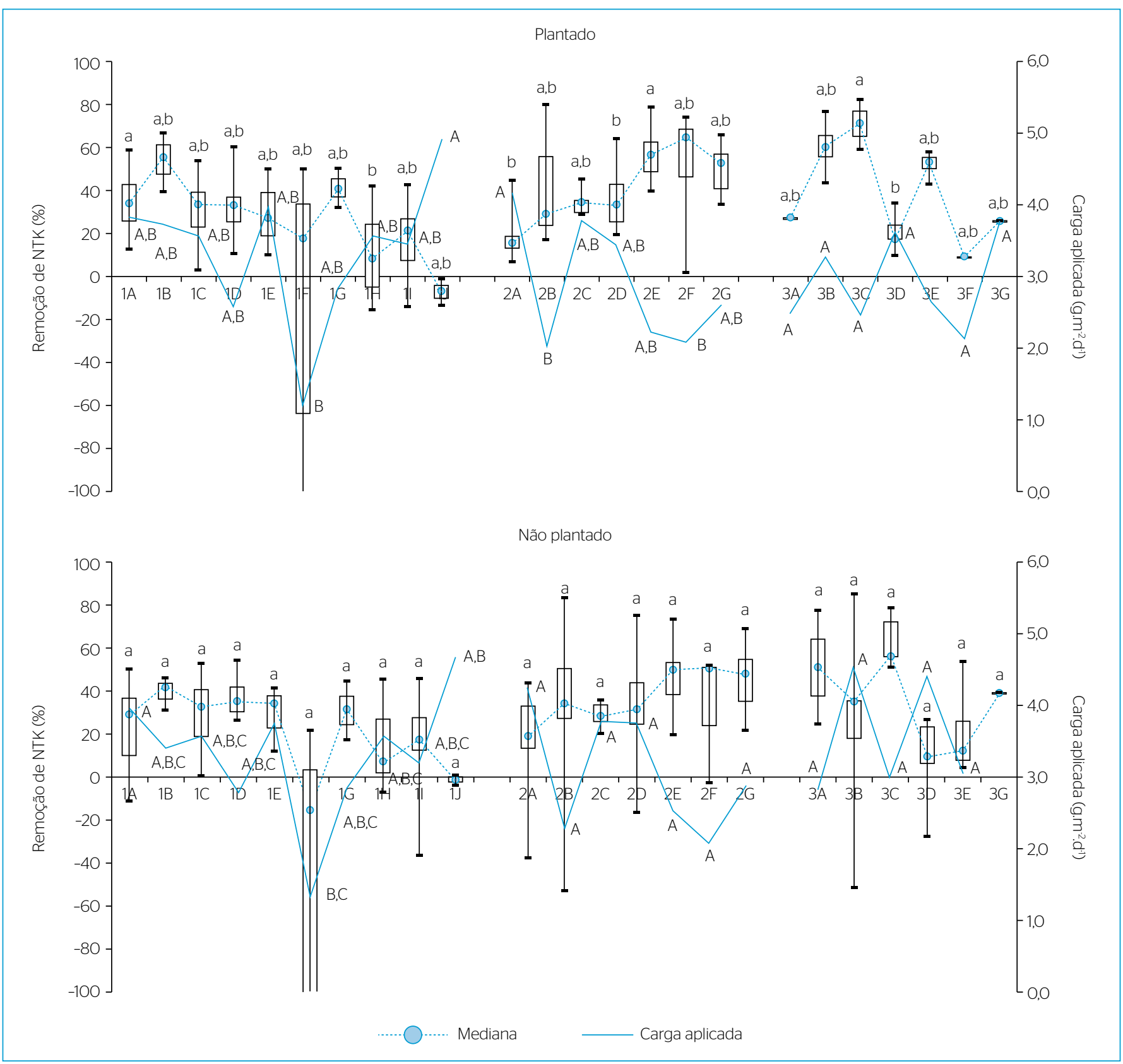

Figura 6 - Box plot das eficiências de remoção de nitrogênio total Kjeldahl nos sistemas alagados construídos plantado e não plantado, durante as subfases (períodos entre cortes das plantas) das três fases. Medianas seguidas pela mesma letra não diferem significativamente (minúsculas para mediana e maiúsculas para cargas aplicadas), pelo teste de Kruskal-Wallis, em nível de $5 \%$ de significância.

desses sistemas por longos períodos de tempo. A carga aplicada e o tipo de pré-tratamento a que o afluente foi submetido parecem ter sido mais decisivos na eficiência dos sistemas em remover nutrientes/poluentes do que o próprio período de operação das unidades, sobretudo no que se refere ao SAC plantado com taboa (Typha latifolia).

O SAC não plantado encontra-se atualmente mais colmatado do que a unidade plantada, havendo prejuízo de seu potencial de remoção de sólidos e nitrogênio em relação ao plantado (comparando as duas últimas fases de monitoramento).

\section{AGRADECIMENTOS}

Os autores agradecem ao Conselho Nacional de Desenvolvimento Científico e Tecnológico (CNPq), à Coordenação de Aperfeiçoamento de Pessoal de Nível Superior (Capes), à Fundação Nacional de Saúde (Funasa), à Financiadora de Inovação e Pesquisa (Finep) e à Fundação de Amparo à Pesquisa de Minas Gerais (Fapemig), pela bolsa e pelo fomento à pesquisa; à COPASA, a disponibilização da área experimental; e ao Departamento de Engenharia Sanitária e Ambiental da Universidade Federal de Minas Gerais (UFMG), a formação do primeiro autor. 


\section{REFERÊNCIAS}

AMERICAN PUBLIC HEALTH ASSOCIATION (APHA); AMERICAN WATER WORKS ASSOCIATION (AWWA); WATER ENVIRONMENT FEDERATION (WEF). (2012). Standard methods for the examination of water and wastewater. 22. ed. Washington: APHA/AWWA/WEF, 1496 p.

BRIX, H. (1997) Do macrophytes play a role in constructed treatment wetlands? Water Science and Technology, v. 35, n. 5 , p. 11-17. https://doi.org/10.1016/SO273-1223(97)00047-4

CASELLES-OSORIO, A.; PUIGAGUT, J.; SEGÚ, E; VAELLO, N.; GRANÉS, F;: GARCIA, D.; GARCIA, J. (2007) Solids accumulation in six full-scale subsurface flow constructed wetlands. Water Research, v. 41, p. 1388-1398. https://doi.org/10.1016/j.watres.2006.12.019

CONLEY, L.M.; DICK, R.I.; LION, L.W. (1991) An assessment of the Root Zone Method of wastewater treatment. Journal of the Water Pollution Control Federation, v. 63, n. 3, p. 239-247.

COSTA, J.F; MARTINS, W.L.P.; SEIDL, M.; VON SPERLING, M. (2015) Role of vegetation (Typha latifolia) on nutrient removal in a horizontal subsurface-flow constructed wetland treating UASB reactor-trickling filter effluent. Water Science and Technology, v. 71, n. 7, p. 1004-1010. https://doi.org/10.2166/wst.2015.055

DORNELAS, F.L:; MACHADO, M.B.; VON SPERLING, M. (2009) Performance evaluation of planted and unplanted subsurfaceflow constructed wetlands for the post-treatment of UASB reactor effluents. Water Science and Technology, v. 60, p. 3025-3033. http:// doi.org/10.2166/wst.2009.743

FU, G.; ZHANG, J.; CHEN, W.; CHEN, Z. (2O13) Medium clogging and the dynamics of organic matter accumulation in constructed wetlands. Ecological Engineering, v. 60, p. 393-398. https://doi. org/10.1016/j.ecoleng.2013.09.012

GAGNON, V.; CHAZARENC, F; KOIV, M.; BRISSON, J. (2O12) Effect of plant species on water quality at the outlet of a sludge treatment wetland. Water Research, v. 46, p. 5305-5315. https://doi.org/10.1016/j. watres.2012.07.007

GARFI, M.; PEDESCOLL, A.; BÉCARES, E.; HIJOSA-VALSERO, M.; SIDRACH-CARDONA, R.; GARCIA, J. (2012). Effect of climatic conditions, season and wastewater quality on contaminant removal efficiency of two experimental constructed wetlands in different regions of Spain. Science of the Total Environment, v. 437, p. 61-67. https://doi.org/10.1016/j.scitotenv.2012.07.087

HUA, G.F.; ZHAO, Z.W.; KONG, J.; GUO; R.; ZENG, Y.T.; ZHAO, L.F.; ZHU, Q.D. (2014) Effects of plant roots on the hydraulic performance during the clogging process in mesocosm vertical flow constructed wetlands. Environmental Science Pollution Research, v. 21, p. 13017-13026. https://doi.org/10.1007/s11356-014-3249-1

KADLEC, R.H.; WALLACE, R.D. (2009) Treatment Wetlands. 2. ed. Florida: CRC Press. $1016 \mathrm{p}$.

LEE, S.Y.; MANIQUIZ, M.C.; CHOI, J.Y.; JEONG, S.M.; KIM, L.H. (2O13) Seasonal nutrient uptake of plant biomass in a constructed wetland treating piggery wastewater effluent. Water Science and Technology, v. 67, n. 6. https://doi.org/10.2166/wst.2013.002
LEE,C.Y.LEE,C.C.:LEE,F.Y.;TSENG,S.K.:LIAO,C.J.(2OO4).Performance of subsurface flow constructed wetland taking pretreated swine effluent under heavy loads. Bioresource Technology, v. 92, p. 173-179. https://doi.org/10.1016/j.biortech.2003.08.012.

LUEDERITZ, V.; ECKERT, E.; LANGE-WEBER, M.; LANGE, A.; GERSBERG, R.M. (2001) Nutrient removal efficiency and resource economics of vertical flow and horizontal flow constructed wetlands. Ecological Engineering, v. 18, p. 157-171. https://doi. org/10.1016/S0925-8574(01)00075-1

MATOS, A.T. (2015) Manual de análise de resíduos sólidos e águas residuárias. Viçosa: Editora da UFV. 150 p.

MATOS, A.T; ABRAHÃO, S.S.; BORGES, A.C; MATOS, M.P. (2O1O) Influência da taxa de carga orgânica no desempenho de sistemas alagados construídos cultivados com forrageiras. Engenharia Sanitária e Ambiental, v. 15, n. 1, p. 83-92. http://dx.doi.org/10.1590/ S1413-41522010000100010

MATOS, A.T; MATOS, M.P. (2017) Disposição de águas residuárias no solo e em sistemas alagados construídos. Viçosa: Editora da UFV. 371 p.

MATOS, M.P; BARRETO, A.B.; VASCONCELLOS, G.R;; MATOS, A.T., SIMÕES, G.F.: VONSPERLING, M. (2017) Difficulties and modifications in the use of available methods for hydraulic conductivity measurements in highly clogged horizontal subsurface flow constructed wetlands. Water Science and Technology, v. 76, n. 7. http://dx.doi.org/10.2166/wst.2017.340

MATOS, M.P.; VON SPERLING, M.; MATOS, A.T; ARANHA, P.R.A.; SANTOS, M.A.; PESSOA, F.D.B.; VIOLA, P.D.D. (2016) Clogging in constructed wetlands: estimation of medium porosity by groundpenetrating radar. In: IWA INTERNATIONAL CONFERENCE ON WETLAND SYSTEMS FOR WATER POLLUTION CONTROL, 15. 2016, Gdansk. Anais... Polônia: IWA Gdansk. v. 2. p. 625-640.

MATOS, M.P.; VON SPERLING, M.; MATOS, A.T., PASSOS, R.G. (2O15) Uso de traçador salino para a avaliação da colmatação e das condições hidrodinâmicas em sistemas alagados construídos de escoamento horizontal subsuperficial. Engenharia Agricola, v. 35, n. 6 , p. 1137-1148. http://dx.doi.org/10.1590/1809-4430-Eng.Agric. v35n6p1137-1148/2015

NAZ, M.; UYANIK, S.; YESILNACAR, I.; SAHINKAYA, E. (2009) Side-by-side comparison of horizontal subsurface flow and free water surface flow constructed wetlands and artificial neural network (ANN) modelling approach. Ecological Engineering, v. 35, p. 1255-1263. http://dx.doi.org/10.1016/j.ecoleng.2009.05.010

NIVALA, J.; KNOWLES, P.; DOTRO, G.; GARCIA, J.; WALLACE, S. (2012) Clogging in subsurface-flow treatment wetlands: Measurement, modeling and management. Water Research, v. 46, p. 1625-1640. http://dx.doi.org/10.1016/j.watres.2011.12.051

PAOLI, A.C.; VON SPERLING, M. (2013) Evaluation of clogging in planted and unplanted horizontal subsurface flow constructed wetlands: solids accumulation and hydraulic conductivity reduction. Water Science and Technology, v. 67, n. 6, p. 1345-1352. https://doi.org/10.2166/wst.2013.008 
PAVINATO,P.S.; ROSOLEM, C.A.(2008) Disponibilidadedenutrientes no solo - Decomposição e liberação de compostos orgânicos de resíduos vegetais. Revista Brasileira de Ciência do Solo, v. 32, p. 911-920. http://dx.doi.org/10.1590/S0100-06832008000300001

RANIERI, E.; YOUNG, T.M. (2012) Clogging influence on metals migration and removal in sub-surface flow constructed wetlands. Journal of Contaminant Hydrology, v. 129-130, p. 38-45. https://doi. org/10.1016/j.jconhyd.2012.01.002

ROUSSEAU, D.P.L.; VANROLLEGHEM, P.A.; DE PAUW, N. (2004) Model-based design of horizontal subsurface flow constructed treatment wetlands: a review. Water Research, v. 38, n. 6, p. 1484-1493. https://doi.org/10.1016/j.watres.2003.12.013

TANNER, C.C:; SUKIAS, J.P.S;; UPSDELL, M.P. (1998) Relationships between loading rates and pollutant removal during maturation of gravel-bed constructed wetlands. Journal of Environmental Quality, v. 27, n. 2, p.448-458.http://dx.doi.org/10.2134/jeq1998.00472425002700020028x

UDEIGWE, T.K.; WANG, J.J. (2010) Biochemical Oxygen Demand Relationships in Typical Agricultural Effluents. Water Air Soil Pollution, v. 213, p. 237-249.

U.S. ENVIRONMENTAL PROTECTION AGENCY (USEPA). (2OOO) Constructed Wetlands Treatment of Municipal Wastewaters. EPA/625/R-99/010, Washington, D.C.: USEPA.

VERA, I.; GARCÍA, J.; SÁEZ, K.; MORAGAS, L.; VIDAL, G. (2011) Performance evaluation of eight years experience of constructed wetland systems in Catalonia as alternative treatment for small communities. Ecological Engineering, v. 37, p. 364-371. https://doi. org/10.1016/j.ecoleng.2010.11.031

VYMAZAL, J. (2009) Horizontal sub-surface flow constructed wetlands Ondrejov and Spalene in the Czech Republic - 15 years of operation. Desalination, v. 246, p. 226-237. https://doi.org/10.1016/j. desal.2008.02.042

WALLACE, S.; KNIGHT, R. (2006) Small-scale constructed wetland treatment systems. In: WALLACE, S. Feasibility, Design Criteria and O\&M Requirements. Alexandria: Water Environment Research Foundation (WERF). 304 p.

ZHANG, M.; ZHAO, L.; MEl, C.; YI, L.; HUA, G. (2014) Effects of Plant Material as Carbon Sources on TN Removal Efficiency and $\mathrm{N}_{2} \mathrm{O}$ Flux in Vertical-Flow-Constructed Wetlands. Water Air Soil Pollution, v. 225, n. 2181, p. 1-11. http://dx.doi.org/10.1007/ s11270-014-2181-9

ZHAO, L.; ZHU, W.; TONG, W. (2009) Clogging processes caused by biofilm growth and organic particle accumulation in lab-scale vertical flow constructed wetlands. Journal of Environmental Science, v. 21, p. 750-757. https://doi.org/10.1016/S10010742(08)62336-0

ZHU, H.; YAN, B.; XU, Y.; GUAN, J.; LIU, S. (2014) Removal of nitrogen and COD in horizontal subsurface flow constructed wetlands under different influent $\mathrm{C} / \mathrm{N}$ ratios. Ecological Engineering, v. 63, p. 58-63. http://dx.doi.org/10.1016/j.ecoleng.2013.12.018 